\title{
THE SPONGE-TIPPED CATHETER-A MODIFICATION OF THE OPEN END CATHETER FOR RECORDING OF MYOMETRIAL ACTIVITY IN VIVO
}

\author{
LARS PH. BENGTSSON \\ Department of Obstetrics and Gynaecology, University of Lund, Sweden
}

(Received 11th September 1967)

Many techniques, especially the intra-uterine rubber balloon, have been used for the recording of uterine contractions in the pregnant and non-pregnant woman. The size of the balloon and its tension, however, greatly influence the results, as Reynolds (1949) has pointed out. Furthermore, it is difficult to maintain exactly the same balloon volume throughout several hours' recording.

Some years ago Hendricks (1964) introduced the 'open end' catheter technique which is rather simple: a thin, fluid-filled polyethylene catheter is introduced into the cavity of the non-pregnant uterus, and its outer end is connected to a recording system. The fluid surface at the inner tip of the catheter acts as a pressure membrane which transmits the pressure in the slit between the anterior and posterior uterine wall to the fluid within the catheter and thence to the recording instrument. In trained hands this technique works very well and it has been successfully used by Hendricks (1964, 1965, 1966), Bengtsson \& Theobald (1966), Bengtsson \& Moawad (1966, 1967), Moawad \& Bengtsson (1967), Cibils (1967) and Coutinho (1967).

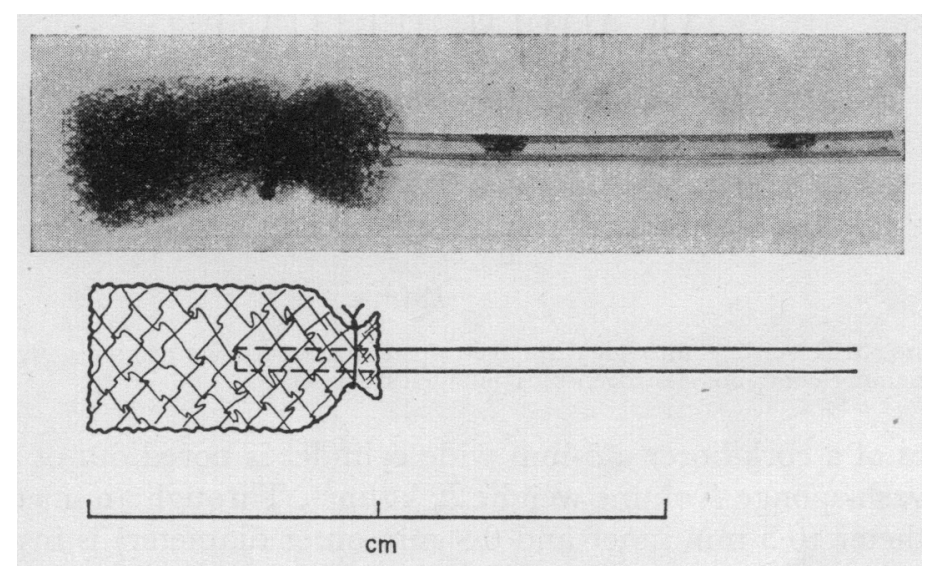

Text-Fig. 1. The sponge-tipped catheter.

However, the open end catheter has certain disadvantages. The fluid surface at the inner tip of the catheter must not slip into the catheter, so the system must be extremely tight. But a tight system is very difficult to create. 
Furthermore, endometrial fragments may occasionally obstruct the inner tip of the catheter, necessitating repeated flushing or continuous counter-pressure.

These disadvantages have been overcome by the sponge-tipped catheter which is prepared as follows:

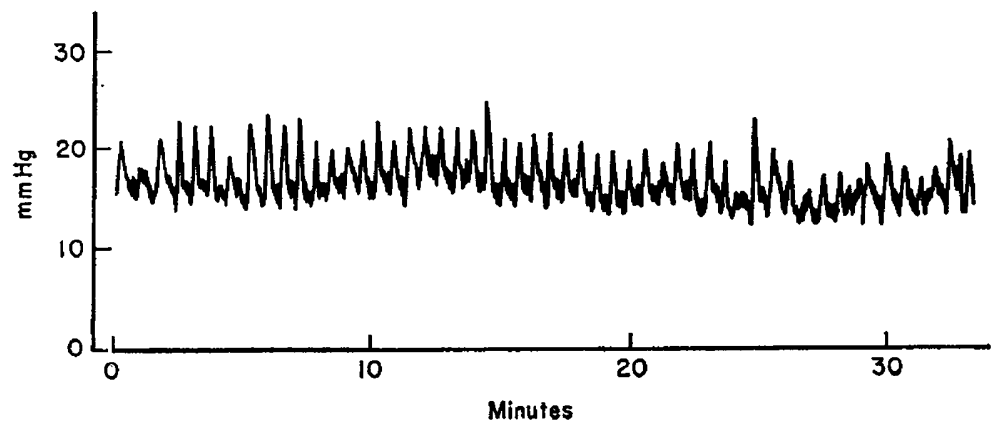

Text-FIG. 2. Recording through a sponge-tipped catheter of the uterine activity in an oestrogen-treated post-menopausal woman.

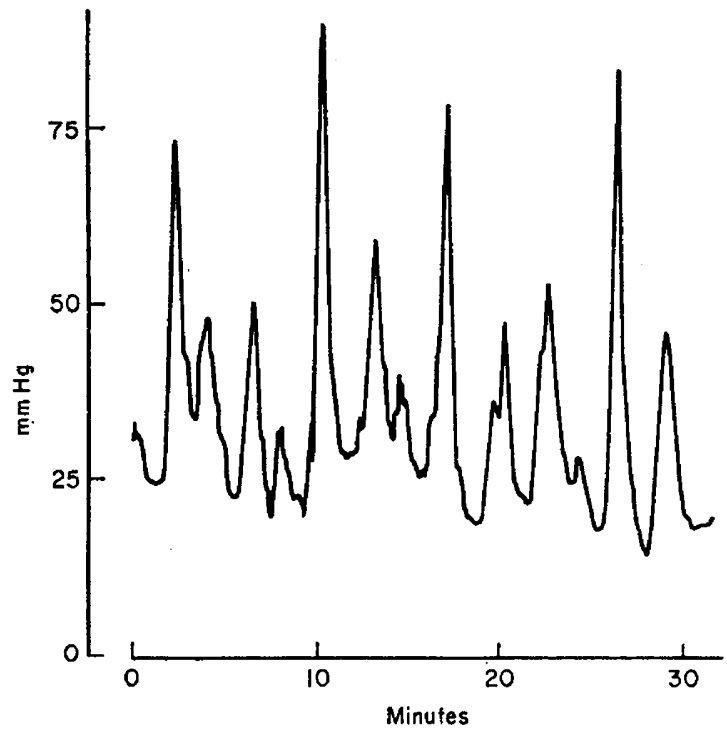

Text-FIG. 3. Recording through a sponge-tipped catheter of the uterine activity in a woman under combined oestrogen plus gestagen treatment.

By means of a cork borer a 5-mm wide cylinder is bored out of an ordinary synthetic wash-sponge (volume weight $22 \mathrm{~kg} / \mathrm{m}^{3}$ ). Through an injection needle a vinyl catheter $(0.5 \mathrm{~mm}$ inner and $0.9 \mathrm{~mm}$ outer diameter) is inserted about $5 \mathrm{~mm}$ into the sponge cylinder and fixed to it by a silk ligature (Text-fig. 1). The cylinder is cut 3 to $5 \mathrm{~mm}$ from the catheter tip. After filling with physiological saline the catheter is inserted into the uterine cavity through a plastic tube of $5 \mathrm{~mm}$ outer diameter, which is then removed. The outer end of the catheter is attached to a recording system, in our experiments a pressure 
transducer (EMT 34, Elema-Schönander, Stockholm), an electro-manometer (Elema-Schönander, Stockholm) and an ink writer (Rika-Denki, Tokyo).

Two or three sponge-tipped catheters with some distance between the ends can be fixed together and introduced simultaneously in the uterus of both women and animals.

The sponge-tipped catheter has been used to our satisfaction in non-pregnant women (examples in Text-figs. 2 and 3); pregnant women before rupture of the membranes, when it was placed between the membranes and the uterine wall; pregnant women after rupture of the membranes; puerperal women; and in non-pregnant rabbits (examples in Text-fig. 4), in which species the gut-like uterine horn causes open end catheters to become rapidly blocked.

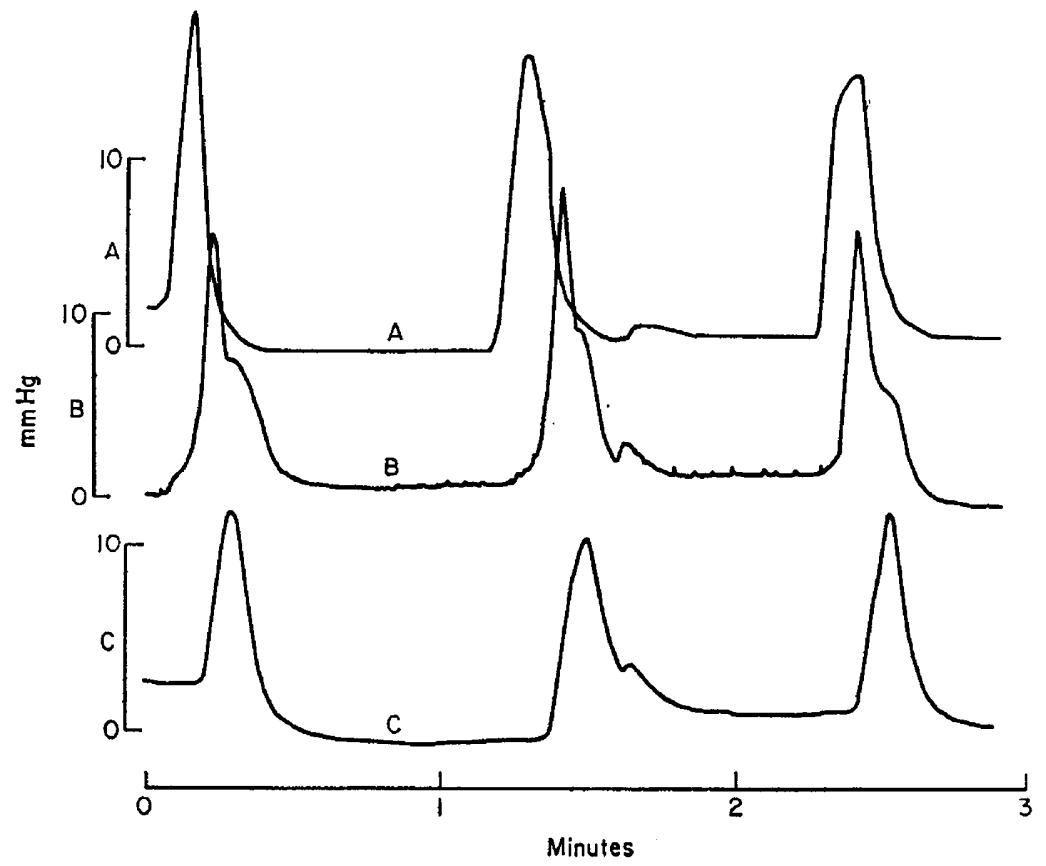

TEXT-FIG. 4. Recording through three sponge-tipped catheters in one horn of an oestrogentreated ovariectomized rabbit. $\mathrm{A}=$ vaginal end; $\mathrm{B}=$ middle portion; $\mathrm{G}=$ tubal end of the horn. Note that the contractions move upwards from the vaginal end.

One important advantage of the sponge-tipped catheter is that its many small channels permit free transmission of the pressure to the catheter tip. The risk of occlusion of all these channels is small. Therefore, flushing of the catheter is rarely needed. We have run many $2-\mathrm{hr}$ recordings without a single flush.

The sponge cylinder has no positive pressure in itself. On the other hand, after compression, a sponge tries to regain its original volume and may then give a slightly negative pressure, increasing with the size of the sponge. Therefore, the sponge cylinder should be as small as possible.

The criticism that has been raised against the open end catheter (and consequently also against the sponge-tipped catheter) that it cannot be used in an 
'open system' such as the uterine cavity, is without substance. The spongetipped catheter readily records the pressure between the moistened palms of the hands (Text-fig. 5)-a situation which definitely represents an open system, i.e. until a pressure is applied, when it closes.

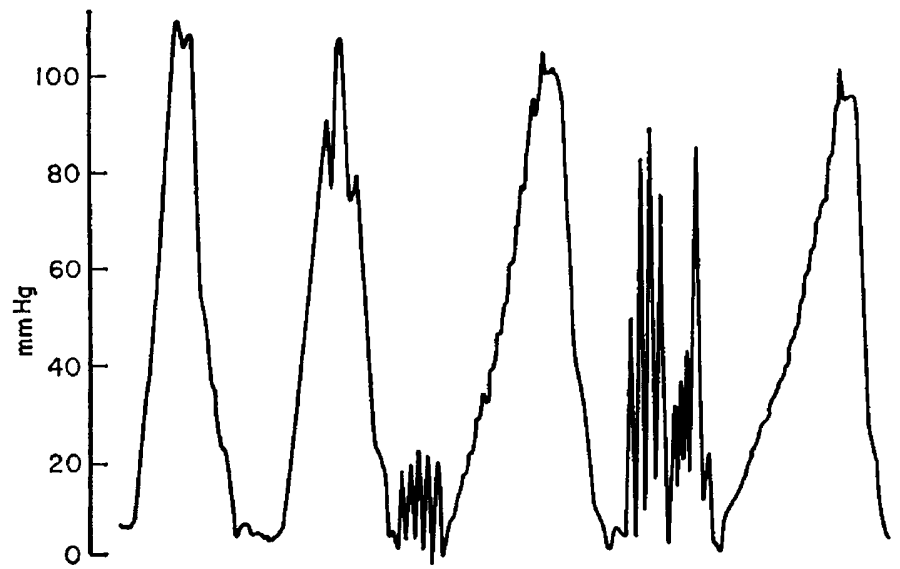

TEXT-FIG. 5. Recording through a sponge-tipped catheter placed between the moistened palms of two hands. Every peak, small or large, slow or rapid, is produced by voluntary increases of the intra-palmar pressure.

In all the instances described we have found this technique reliable and necessitating a minimum of training. It can also be used in other organs with a real or potential lumen, e.g. oesophagus, urethra, urinary bladder and rectum.

\section{REFERENCES}

Bengtsson, L. Ph. \& Moawad, A. H. (1966) Lippes' loop and myometrial activity. Lancet, i, 146.

Bengtsson, L. Ph. \& Moawad, A. H. (1967) The effect of Lippes' loop on human myometrial activity. Am. 7. Obstet. Gynec. 98, 957.

Bengtsson, L. Ph. \& Theobald, G. W. (1966) The effects of oestrogen and gestagen on the nonpregnant human uterus. F. Obst. Gynaec. Br. Commonw. 73, 273.

CrBiLs, L. A. (1967) Contractility of the nonpregnant human uterus. Obstet. Gynec., N.Y. 30, 441.

Coutinho, E. M. (1967) Uterine activity in non-pregnant women. Proc. 8th int. Conf. plann. Parent. Santiago, p. 432. IPPF, London.

HENDRICKs, C. H. (1964) A new technique for the study of motility in the non-pregnant human uterus. 7. Obstet. Gynaec. Br. Commonw. 71, 712.

Hendricks, C. H. (1965) Activity patterns in the non-pregnant human uterus. In: Muscle. Eds. W. M. Paul, E. E. Daniels, E. M. Kay and G. Monckton. Pergamon Press, New York.

Hendricks, C. H. (1966) Inherent motility patterns and response characteristics of the nonpregnant human uterus. Am. 7. Obstet. Gynec. 96, 824.

Moawad, A. H. \& Bengtsson, L. Pr. (1967) In vivo studies of the motility patterns of the nonpregnant human uterus. I. The normal menstrual cycle. Am. F. Obstet. Gynec. 98, 1057.

Reynolds, S. R. M. (1949) Physiology of the uterus. Hoeber, New York. 